\title{
Strong Tunneling in the Single-Electron Box
}

\author{
Jürgen König and Herbert Schoeller \\ Institut für Theoretische Festkörperphysik, Universität Karlsruhe, 76128 Karlsruhe, Germany
}

(January 7, 2014)

\begin{abstract}
We study strong tunneling (i.e. transmission $h / e^{2} R_{T} \gg 1$ ) in the single-electron box with many transverse modes at zero temperature. We develop a new renormalization group method which includes all charge states and requires no initial or final energy cutoff. We determine the ground-state energy, the average charge and the renormalized charging energy. The covered range for the coupling constant and the gate voltage is much increased in comparison to recent perturbative approaches, poor man scaling methods and Quantum Monte Carlo simulations. We reach the regime where Coulomb blockade become practically unobservable.
\end{abstract}

73.23.Hk,73.40.Gk,73.30.Rw

Introduction. Metallic nanostructures with junctions of low capacitance show quantization of electric charge and Coulomb blockade phenomena due to large charging energy [1] 3]. The simplest system in which one can see Coulomb blockade effects is the single-electron box. Electrons enter or leave a small metallic island via a tunnel junction with many transverse channels. The junction is characterized by a resistance $R_{T}$ and a capacitance $C$. The charge on the island is controlled by a capacitively (with $C_{G}$ ) coupled external voltage $V_{G}$. In the "weak tunneling" regime, i.e., when the transmission $\mathcal{T}=h / e^{2} R_{T}$ of the barrier is much less than unity, the average charge of the box can be described within the "orthodox theory" [1] which treats tunneling in lowest order perturbation theory (golden rule). This means that the energy for charge $N$ on the island is given by the classical expression

$$
E_{N}\left(n_{x}\right)=E_{C}\left(N-n_{x}\right)^{2},
$$

with $E_{C}=e^{2} / 2\left(C+C_{G}\right)$ and $n_{x}=C_{G} V_{G}$. At zero temperature, the ground-state energy as function of $n_{x}$ is the periodic continuation of the parabola $E_{0}\left(n_{x}\right)$ for $-1 / 2<n_{x}<1 / 2$. Furthermore, the average charge, given by the periodic continuation of

$$
\langle N\rangle\left(n_{x}\right)=n_{x}-\frac{1}{2 E_{C}} \frac{\partial E_{0}}{\partial n_{x}}\left(n_{x}\right),
$$

increases in steps at half-integer values of $n_{x}$.

In this letter, we discuss the fundamental question how quantum fluctuations influence Coulomb blockade phenomena at zero temperature, i.e., we analyze the smearing of $E_{0}\left(n_{x}\right)$ and $\langle N\rangle\left(n_{x}\right)$ in the whole regime from weak to strong tunneling up to $\mathcal{T} \sim 50$ (in recent experiments $\mathcal{T} \sim 33$ was achieved (4]). Another quantity of recent interest is the second derivative of the energy $E_{0}$,

$$
E_{C}^{*}\left(n_{x}\right)=\frac{1}{2} \frac{\partial^{2} E_{0}}{\partial n_{x}^{2}}\left(n_{x}\right)
$$

Perturbative approaches [5] in $\alpha=\mathcal{T} /\left(4 \pi^{2}\right)$ up to $\alpha^{3}$ [6] can describe $E_{C}^{*}(0)$ up to $\mathcal{T} \sim 15$. However, within this approach, the range of allowed values for $\mathcal{T}$ decreases considerably for $n_{x} \rightarrow 1 / 2$, and the average charge turns out to be divergent at $n_{x}=1 / 2$.

Therefore, nonperturbative approaches have been developed to describe the system near the degeneracy point of adjacent charge states either within a "non-crossing" expansion scheme [9] or using an effective two-state system 8, 10 12]. But for $\mathcal{T} \gtrsim 1$ the broadening $\hbar /\left(R_{T} C\right) \sim$ $\mathcal{T} E_{C} / \pi$ of the charge states is of the same order as the energy gap $E_{C}$, i.e., all charge states become important. And even for weaker coupling the results from a renormalization group (RG) treatment [8,10] depend on both a high-energy and a low-energy cutoff. The first one, of the order of the charging energy, accounts for the neglected higher charge states. The second one is provided by the dominant low-energy scale, energy gap or temperature. As a result, quantitative statements depend on adjustable parameters.

For very strong coupling, $\mathcal{T} \gg 1$, instanton methods have been developed, which predict an exponential decrease of Coulomb blockade phenomena: $E_{C}^{*}(0) / E_{C} \sim$ $\mathcal{T}^{\eta} \exp (-\mathcal{T} / 2)$, 13, 14. However, the exponent $\eta$ is still controversial. Poor man scaling methods in $1 / \mathcal{T}$ reveal a strong renormalization of $\mathcal{T}$ to smaller values [10,15. This analysis is performed in the phase representation for the topological sector with winding number zero. However, for decreasing $\mathcal{T}$ all winding numbers become important. Consequently, a quantitative matching to the two-charge state approximation is not possible 16.

Even recent quantum Monte Carlo (QMC) simulations [6, 15, 17, 18] have led to different results for $E_{C}^{*}(0)$ in the regime $\mathcal{T}>15$. Furthermore, the error bars and the computing time are very large for $\mathcal{T} \gg 1$ and/or $n_{x} \rightarrow$ $1 / 2$ and extrapolation to zero temperature in order to describe ground state properties becomes difficult. As a consequence, a large gap still remains between the small and large $\mathcal{T}$-regions, the latter being even controversial.

It is the purpose of this letter to present an independent, real-time RG-method which covers self-consistently level-renormalization and -broadening together with vertex corrections, and includes all charge states without the need of initial or final energy cutoffs. In contrast to QMC simulations our theory works also at zero temperature. For $E_{C}^{*}(0)$ we find good agreement with the QMC sim- 
ulation of Refs. [6, 15]. We also find agreement with the exponential behavior of $E_{C}^{*}(0)$ for $\mathcal{T} \gtrsim 20$, as predicted by the controversial instanton results in Refs. [13,14 but with a different preexponent. We calculate $\langle N\rangle\left(n_{x}\right)$ for all $n_{x}$ and predict that for $\mathcal{T}>27$ charge quantization will be suppressed such that the deviation from a straight line is less than $1 \%$. We find that the slope of $\langle N\rangle$ at $n_{x}=1 / 2$ is always infinite.

Model. The system is modeled by the Hamiltonian $H=H_{0}+H_{T}$, in which

$$
H_{0}=\sum_{k n} \epsilon_{k n} a_{k n}^{\dagger} a_{k n}+\sum_{q n} \epsilon_{q n} c_{q n}^{\dagger} c_{q n}+\sum_{N} E_{N}|N\rangle\langle N|
$$

describes the box without tunneling. The operators $a^{(\dagger)}$ and $c^{(\dagger)}$ annihilate (create) electrons in the lead and island electrode, respectively. The tunneling coupling is given by

$$
H_{T}=\sum_{N} \sum_{\sigma= \pm} g_{N}^{\sigma}|N+\sigma\rangle\langle N| J_{\sigma}
$$

with $J_{-}=\sum_{k q n} T_{k q}^{n} a_{k n}^{\dagger} c_{q n}$ and $J_{+}=J_{-}^{\dagger}$. The channel number $n=1, \ldots, N_{c h}$ accounts for both the transverse wave number and the spin and is conserved during a tunnel process. We assume here a wide metallic junction with a large number of transverse channels. The matrix elements $T_{k q}^{n}$ are assumed to be independent of the states $k$ and $q$. They are related to the tunnel resistance by $1 / R_{T}=\left(2 \pi e^{2} / \hbar\right) \sum_{n} N_{L}(0) N_{I}(0)\left|T^{n}\right|^{2}$, where $N_{L / I}(0)$ are the density of states of the lead and the island. The factor $g_{N}^{\sigma}$ describes the relative strength of the tunnel coupling. At the beginning, we have $g_{N}^{\sigma} \equiv 1$ for all $N$ and $\sigma$. But under the renormalization group procedure, the coupling will indeed depend on the charge state $N$ and on $\sigma$. We define $\alpha_{N}=\alpha g_{N}^{+} g_{N+1}^{-}$.

Renormalization group procedure. The invariant quantity under the RG is the S-matrix $S=$ $T \exp \left(-i \int d t H(t)\right)$. The idea of the RG is to start with some high-energy cutoff, reducing it by integrating out the corresponding degrees of freedom, and end up with an effective low-energy theory. All the energies and coupling constants will be renormalized, and, simultaneously, new terms which are not present in the original Hamiltonian may be generated. We will proceed in three steps. (i) We introduce a time cutoff $t_{c}$. (ii) The cutoff is increased $t_{c} \rightarrow t_{c}+d t_{c}$, thus flowing from $t_{c}^{0}$ to $t_{c}^{f}$. The invariance condition for the S-matrix demands

$$
\left.T e^{-i \int d t H(t)}\right|_{t_{c}}=\left.T e^{-i \int d t H^{\prime}(t)}\right|_{t_{c}+d t_{c}}
$$

for each RG step. (iii) We take $t_{c}^{0} \rightarrow 0$ and $t_{c}^{f} \rightarrow \infty$.

(i) The S-matrix reads in interaction picture $S=$ $T \exp \left(-i \int d t H_{0}\right) T \exp \left(-i \int d t H_{T}(t)_{I}\right)$ in which $H_{T}(t)_{I}$ is the tunnel part of the Hamiltonian in interaction picture with respect to $H_{0}$. The expansion of the second exponential yields

$$
S=T e^{-i \int d t H_{0}} \sum_{n=0}^{\infty}(-i)^{n} \int_{t_{1}>t_{2}>\ldots>t_{n}} H_{1} \ldots H_{n},
$$

where $H_{i} \equiv H_{T}\left(t_{i}\right)_{I}$. Next, we perform a normal ordering of the fermion operators $c^{(\dagger)}$ and $a^{(\dagger)}$ involved in $H_{i}$

$$
\begin{aligned}
& H_{1}=: H_{1}: \\
& H_{1} H_{2}=: H_{1} H_{2}:+\overparen{H}_{1} H_{2} \\
& H_{1} H_{2} H_{3}=: H_{1} H_{2} H_{3}:+\overparen{H}_{1} H_{2} H_{3} \\
& +{\overparen{H_{1} H_{2} H_{3}}}+H_{1} \overparen{H}_{2} H_{3}
\end{aligned}
$$

where : . . : stands for normal ordering and $\overparen{H}_{1} H_{2}$ represents contractions of all the Fermi operators $c^{(\dagger)}$ and $a^{(\dagger)}$ in $H_{1}$ and $H_{2}$ with the relative time $t_{1}-t_{2}$. In principle the two Fermi operators of $H_{i}$ may be contracted with Fermi operators of different $H_{i^{\prime}}$ 's. For a large number of channels, $N_{c h} \rightarrow \infty$, however, these contributions are negligible. The only combination of contractions which enter here is (at zero temperature)

$$
\left\langle J_{ \pm}\left(t_{1}\right) J_{\mp}\left(t_{2}\right)\right\rangle_{e q}=-\frac{\alpha}{\left(t_{1}-t_{2}\right)^{2}}+i \alpha \pi \delta^{\prime}\left(t_{1}-t_{2}\right) .
$$

We now introduce a cutoff $t_{c}$ by multiplying the right hand side of Eq. (11) with $\Theta\left(\left|t_{1}-t_{2}\right|-t_{c}\right)$. Consequently, via Eqs. (7)-(10), the S-matrix is well-defined with a sharp cutoff $t_{c}$.

(ii) The increase of the cutoff $t_{c}^{\prime}=t_{c}+d t_{c}$ leads to correction terms $\mathrm{H}_{1} \mathrm{H}_{2}, \mathrm{H}_{1} \mathrm{H}_{2} \mathrm{H}_{3}$, etc. The cross indicates that the time difference is between $t_{c}$ and $t_{c}^{\prime}$. We interpret such many-time objects as clusters with one time argument for the time ordering (we choose $t_{2}$ ). It is possible to reexponentiate the sum of terms and correction terms. The renormalized exponent reads

$$
\begin{aligned}
& -i \int H_{1}^{\prime}=-i \int H_{1}+(-i)^{2} \int_{t_{1}>t_{2}} \stackrel{H_{1}^{*}}{H_{2}}
\end{aligned}
$$

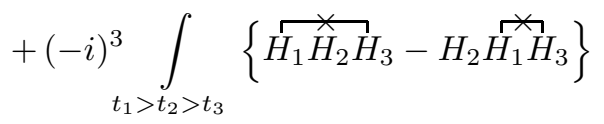

plus higher-order correction terms which we truncate. This is the central equation since it determines the renormalization of all quantities. The second term (propagator renormalization) can be absorbed in a change of the energies, while the third term (vertex corrections) renormalizes the coupling strength.

(iii) The limit $t_{c}^{0} \rightarrow 0$ is possible if we take into account all charge states. There are no divergences like in a twostate approximation. After performing the limit $t_{c}^{f} \rightarrow \infty$ 
we end up with an effective theory for the charge degrees of freedom without any contractions.

To present the results, we introduce the abbreviations $l=\ln \left(t_{c} / t_{c}^{0}\right), \Delta_{N}=E_{N+1}-E_{N}, \bar{E}_{N}=E_{N} t_{c}$, and $\bar{\Delta}_{N}=$ $\Delta_{N} t_{c}$. The RG equations, then, are

$$
\begin{aligned}
& \frac{d \bar{E}_{N}}{d l}=\bar{E}_{N}+i\left(\alpha_{N-1} e^{i \bar{\Delta}_{N-1}}+\alpha_{N} e^{-i \bar{\Delta}_{N}}\right) \\
& \frac{d \bar{\Delta}_{N}}{d l}=\bar{\Delta}_{N}+i\left(\alpha_{N} e^{i \bar{\Delta}_{N}}+\alpha_{N+1} e^{-i \bar{\Delta}_{N+1}}\right. \\
& \left.-\alpha_{N} e^{-i \bar{\Delta}_{N}}-\alpha_{N-1} e^{i \bar{\Delta}_{N-1}}\right) \\
& \frac{d \alpha_{N}}{d l}=\alpha_{N}\left\{2 \alpha_{N+1} \frac{e^{-i \bar{\Delta}_{N+1}}-e^{-i \bar{\Delta}_{N}}}{-i\left(\bar{\Delta}_{N+1}-\bar{\Delta}_{N}\right)}\right. \\
& +2 \alpha_{N-1} \frac{e^{i \bar{\Delta}_{N}}-e^{i \bar{\Delta}_{N-1}}}{i\left(\bar{\Delta}_{N}-\bar{\Delta}_{N-1}\right)} \\
& -\alpha_{N} e^{i \bar{\Delta}_{N}}-\alpha_{N+1} e^{-i \bar{\Delta}_{N+1}} \\
& \left.-\alpha_{N} e^{-i \bar{\Delta}_{N}}-\alpha_{N-1} e^{i \bar{\Delta}_{N-1}}\right\} \text {. }
\end{aligned}
$$

Discussion of the $R G$ equations. We note the following:

- All energies become complex, which is related to the broadening of the levels.

- The coupling strength $\alpha_{N}$ and the difference $\Delta_{N+1}-$ $\Delta_{N}$, which are initially $\alpha$ and $2 E_{C}$, respectively, evolve different for different charge states $N$.

- The RG equations contain all terms from perturbation theory up to $\mathcal{O}\left(\alpha^{2}\right)$.

- Eqs. (14) and (15) include also all terms of the poor man's scaling. If one considers for $n_{x} \approx 1 / 2$ only two charge states $N=0,1$, and expands all exponentials $\exp \left( \pm i \bar{\Delta}_{0}\right) \approx 1 \pm i \bar{\Delta}_{0}$, Eqs. (14) and (15) reduce to the well-known formulas $d \bar{\Delta}_{0} / d l=\Delta_{0}\left(1-2 \alpha_{0}\right)$ and $d \alpha_{0} / d l=$ $-2 \alpha_{0}^{2}$, with solutions $\Delta_{0}(l) / \Delta_{0}(0)=\alpha_{0}(l) / \alpha_{0}(0)=$ $\left[1+2 \alpha_{0}(0) l\right]^{-1}$ with $\alpha_{0}(0)=\alpha$.

Results. In Figs. 田-3, we show $E_{0},\langle N\rangle$, and $E_{C}^{*}$ as function of $n_{x}$ up to $\alpha=0.5(\mathcal{T} \sim 20)$ and compare with QMC data from Refs. [6, 18]. (In the numerics we had to choose the initial cutoff $t_{c}^{0}$ much smaller than $0.1 E_{C}^{-1}$. Here, we chose $0.01 E_{C}^{-1}$.) As expected we find a gradual flattening of the ground-state energy with increasing coupling and a rounding of the average charge towards a linear function. The average charge $\langle N\rangle\left(n_{x}\right)$ agrees well with available QMC data for $\alpha \leq 0.3$, except for $n_{x} \gtrsim 0.4$. This deviation can be traced back to a finite temperature used in the QMC simulations [19]. ¿From our results we conclude: (i) For $\mathcal{T} \sim 27$, the shape of $\langle N\rangle\left(n_{x}\right)$ is nearly indistinguishable from a straight line within an error of $1 \%$. (ii) The slope of $\langle N\rangle$ at $n_{x}=1 / 2$ is infinite, i.e., there is always a very small crossover region around the degeneracy point showing a deviation from a straight line. This is explicitly shown in Fig. 3 for the renormalized charging energy which diverges at $n_{x}=1 / 2$. However, this region is so small that it can hardly be identified experimentally. (iii) The renormalized charging energy at $n_{x}=0$ decreases with increasing coupling. This reveals another indication for the washing out of charge quantization and is shown in more detail in Fig. 1 using a logarithmic scale for $E_{C}^{*}(0)$ as function of the coupling $\alpha$. Here we find very good agreement of our results with perturbation theory [5, 5] up to $\alpha \sim 0.4$ $(\mathcal{T} \sim 15)$ and with the QMC data of Ref. [6]. Fig. 5 shows the comparism to other QMC simulations. Including error bars, our data are consistent with the QMC data of Ref. 115] (the authors report that the saturation for the largest $\mathcal{T}$ is still unclear [20]), but are slightly lower than those of Ref. [17, and higher than those of Ref. [18]. For $\mathcal{T}>25$ no QMC data are available yet but the numerical solution of the RG-equations (13)-(15) is stable until $\mathcal{T} \sim 50$. For $\mathcal{T} \gtrsim 20$ our data are consistent with the exponential behavior $E_{C}^{*}(0) / E_{C} \sim \mathcal{T}^{\eta} \exp (-\mathcal{T} / 2)$ predicted by instanton techniques [13,14] but with $\eta=6.5$ rather than $\eta=2$ or 3 .

Summary. We have investigated strong tunneling in the single-electron box with the help of a new, renormalization group method beyond the perturbative regime. All charge states are included and, therefore, all results are cut-off independent. We analyzed the observability of charging effects at zero temperature by computing the average charge as function of the gate voltage. We found a deviation of less than $1 \%$ from a straight line for $\mathcal{T}>27$ and an infinite slope at the degeneracy point. Furthermore we compared our results to contradicting QMC simulations and instanton techniques.

We acknowledge useful discussions with G. Göppert, H. Grabert, C.P. Herrero, G. Schön, and A.D. Zaikin. This work was supported by the "Deutsche Forschungsgemeinschaft" as part of "SFB 195".

[1] D.V. Averin and K.K. Likharev, in Mesoscopic Phenomena in Solids, ed. B.L. Altshuler et al. (Elsevier, 1991).

[2] Single Charge Tunneling, NATO ASI Series 294, H. Grabert and M.H. Devoret, eds., (Plenum Press, 1992).

[3] G. Schön, Single-Electron Tunneling, in Quantum Processes and Dissipation (VCH Publishers, 1998), T. Dittrich et al., eds., Chapter 3, p.149-212.

[4] P. Lafarge et al., Z. Phys. B 85, 327 (1991); P. Joyez, V. Bouchiat, D. Esteve, C. Urbina, and M.H. Devoret, Phys. Rev. Lett. 79, 1349 (1997); D. Chouvaev, L.S. Kuzmin, D.S. Golubev, and A.D. Zaikin, cond-mat/9803015.

[5] H. Grabert, Phys. Rev. B 50, 17364 (1994).

[6] G. Göppert, H. Grabert, N. Prokof'ev, and B. Svistunov, cond-mat/9802248.

[7] J. König, H. Schoeller, and G. Schön, Phys. Rev. Lett. 78, 4482 (1997); and in cond-mat/9801214.

[8] K.A. Matveev, Sov. Phys. JETP 72, 892 (1991), [Zh. 
Eksp. Teor. Fiz. 99, 1598 (1991)].

[9] D.S. Golubev and A.D. Zaikin, Phys. Rev. B 50, 8736 (1994).

[10] G. Falci, G. Schön, and G.T. Zimanyi, Phys. Rev. Lett. 74, 3257 (1995).

[11] H. Schoeller and G. Schön, Phys. Rev. B 50, 18436 (1994); Physica B 203, 423 (1994).

[12] J. König, H. Schoeller, and G. Schön, Europhys. Lett. 31, 31 (1995); and in Quantum Dynamics of Submicron Structures, eds. H. A. Cerdeira et al., NATO ASI Series E, Vol. 291 (Kluwer, 1995), p.221.

[13] S.V. Panyukov, A.D. Zaikin, Phys. Rev. Lett. 67, 3168 (1991).

[14] X. Wang, H. Grabert, Phys. Rev. B 53, 12621 (1996).

[15] W. Hofstetter and W. Zwerger, Phys. Rev. Lett. 78, 3737 (1997).

[16] D.S. Golubev, J. König, H.Schoeller, G. Schön, and A.D. Zaikin, Phys. Rev. B 56, 15782 (1997).

[17] X. Wang, R. Egger, and H. Grabert, Europhys. Lett. 38, 545 (1997).

[18] C.P. Herrero, G. Schön, and A.D. Zaikin, preprint.

[19] C.P. Herrero, private communication.

[20] W. Hofstetter, private communication.

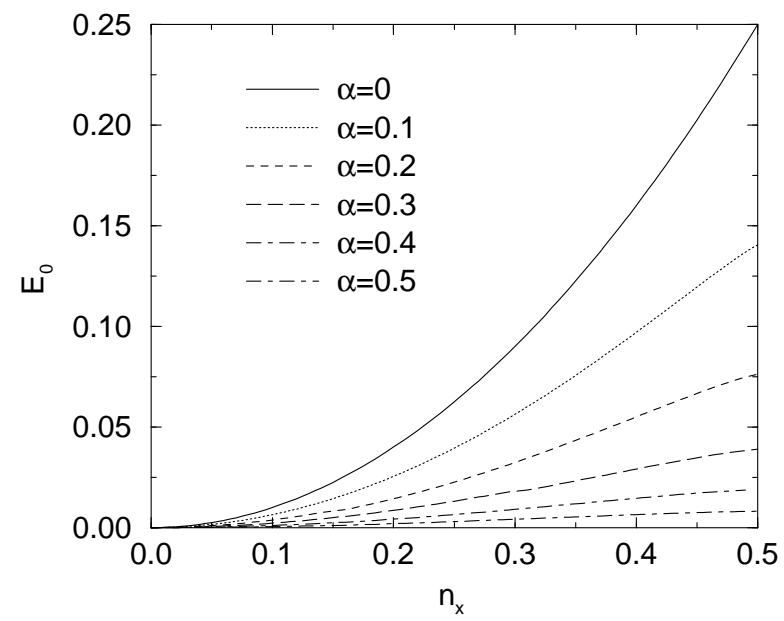

FIG. 1. Ground-state energy as function of $n_{x}$.

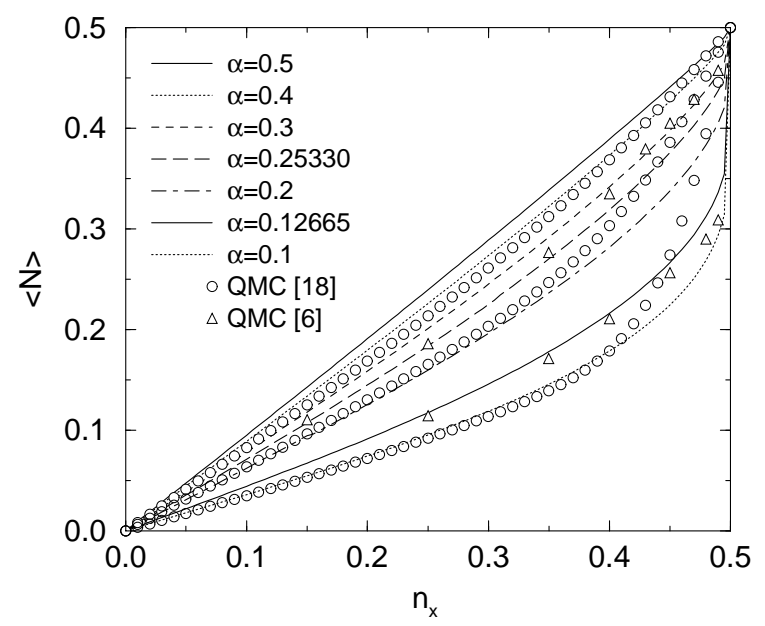

FIG. 2. Average charge as function of $n_{x}$. The step at $n_{x}=1 / 2$ is rounded for increasing $\alpha$, approaching more and more a straight line. The circles are QMC data from Ref. 18 for $\alpha=0.3,0.2,0.1$. The triangles are QMC data from Ref. [a] for $\alpha=0.25330,0.12665$.

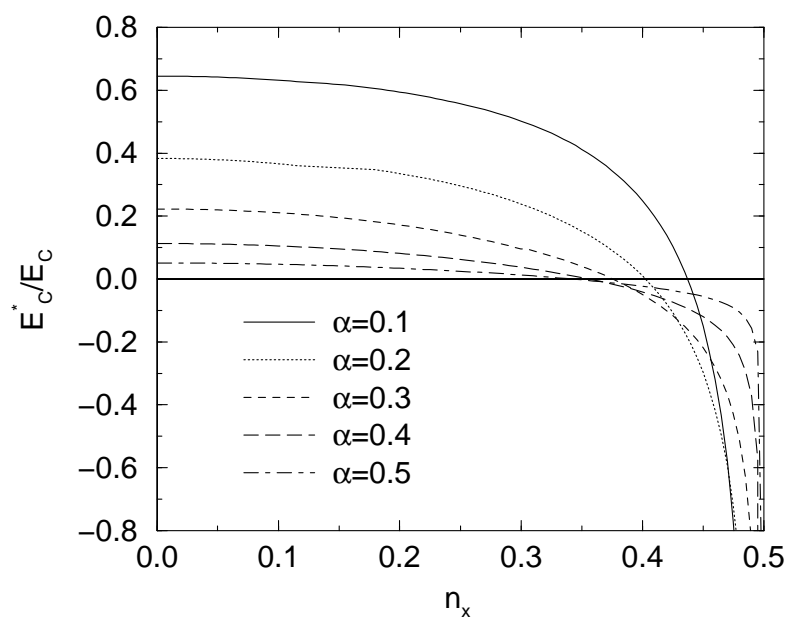

FIG. 3. $E_{C}^{*}$ as a function of $n_{x}$. For $n_{x} \rightarrow 1 / 2$ it diverges.

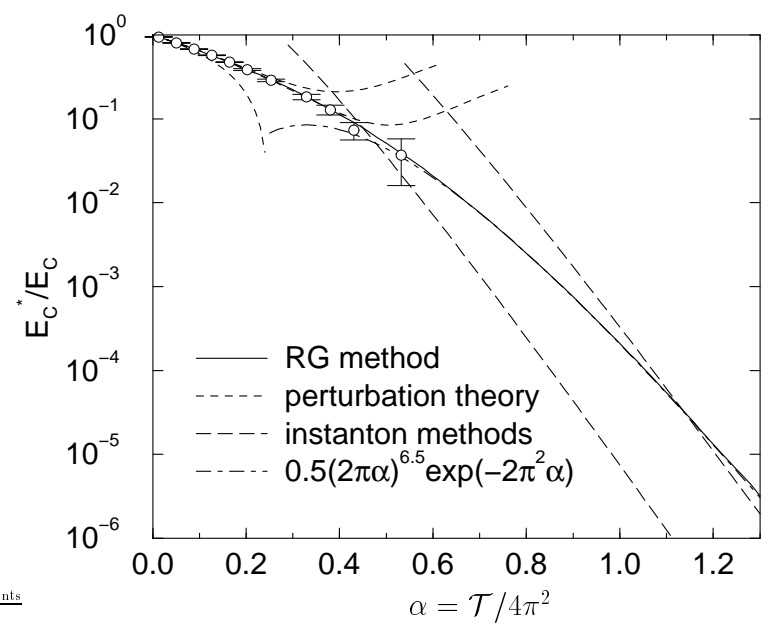

FIG. 4. $E_{C}^{*}$ as function of $\alpha$ for $n_{x}=0$. Solid line: renormalization group method described in this letter. Dashed lines: perturbation theory of order $\alpha, \alpha^{2}$, and $\alpha^{3}$ from Refs. [5, 6. Long-dashed lines: large- $\alpha$ expansions from Refs. 14 (upper curve) and 13] (lower curve). Dot-dashed line: For $\alpha \gtrsim 0.5$ our data are consistent with $0.5(2 \pi \alpha)^{6.5} \exp \left(-2 \pi^{2} \alpha\right)$. Circles: QMC data from Ref. [6]. 


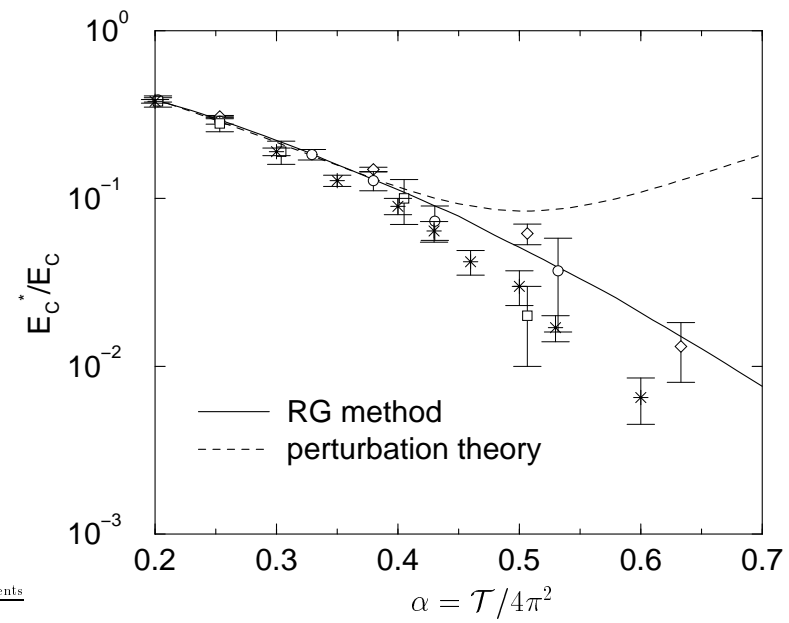

FIG. 5. $E_{C}^{*}$ as function of $\alpha$ for $n_{x}=0$. Solid line: renormalization group method described in this letter. Dashed lines: perturbation theory of order $\alpha^{3}[6]$. QMC data from Ref. 17] $(\diamond)$, [6] (०), 15] ( $\square)$ and 18] $(*)$. 